\title{
Article \\ Thermal Conductance of Graphene-Titanium Interface: A Molecular Simulation
}

\author{
Bingxian Ou ${ }^{1,2}$, Junxia Yan ${ }^{1}$, Qinsheng Wang ${ }^{2}$ and Lixin Lu ${ }^{1, *}$ \\ 1 School of Mechanical Engineering, Jiangnan University, Wuxi 214122, China; obxobx@163.com (B.O.); \\ yjx@jiangnan.edu.cn (J.Y.) \\ 2 Special Equipment Safety Supervision Inspection Institute of Jiangsu Province, National Graphene Products \\ Quality Inspection and Testing Center (Jiangsu), Wuxi 214174, China; wqs@wxtjy.com \\ * Correspondence: lulx@jiangnan.edu.cn
}

Citation: Ou, B.; Yan, J.; Wang, Q.; Lu,

L. Thermal Conductance of

Graphene-Titanium Interface: A

Molecular Simulation. Molecules 2022

27, 905. https://doi.org/10.3390/

molecules27030905

Academic Editor: Teobald Kupka

Received: 29 December 2021

Accepted: 23 January 2022

Published: 28 January 2022

Publisher's Note: MDPI stays neutral with regard to jurisdictional claims in published maps and institutional affiliations.

Copyright: (C) 2022 by the authors. Licensee MDPI, Basel, Switzerland. This article is an open access article distributed under the terms and conditions of the Creative Commons Attribution (CC BY) license (https:// creativecommons.org/licenses/by/ $4.0 /)$.

\begin{abstract}
Titanium is a commonly used material in aviation, aerospace, and military applications, due to the outstanding mechanical properties of titanium and its alloys. However, its relatively low thermal conductivity restricts its extended usage. The use of graphene as a filler shows great potential for the enhancement of thermal conductivity in titanium-based metal-matrix composites (MMCs). We used classical molecular dynamics (MD) simulation methods to explore the thermal conductance at the titanium-graphene (Ti/Gr) interface for its thermal boundary conductance, which plays an important role in the thermal properties of Ti-based MMCs. The effects of system size, layer number, temperature, and strain were considered. The results show that the thermal boundary conductance $(\mathrm{TBC})$ decreases with an increasing layer number and reaches a plateau at $\mathrm{n}=5$. TBC falls under tensile strain and, in turn, it grows with compressive strain. The variation of TBC is explained qualitatively by the interfacial atomic vibration coupling factor. Our findings also provide insights into ways to optimize future thermal management based on Ti-based MMCs materials.
\end{abstract}

Keywords: thermal boundary conductance; titanium-graphene; molecular dynamics

\section{Introduction}

Compared with other metals, titanium and its alloy derivatives are known for their excellent physical properties, stable chemical properties, and great biocompatibilities and are widely applied in aerospace [1], the military [2], automobile manufacturing [3], bone substitute manufacturing $[4,5]$, and other fields. Titanium has a high strength-to-mass ratio. For example, titanium is $60 \%$ denser than aluminum but more than twice as strong as the most commonly used aluminum alloy [6]. The tensile strength of some titanium alloys can even reach $1400 \mathrm{MPa}[1,7]$. The relatively high melting point (more than $1650^{\circ} \mathrm{C}$ ) makes titanium useful as a refractory metal. In addition, titanium and its alloys have good corrosion resistance $[8,9]$. However, the vital drawbacks of titanium and its alloys cannot be ignored. It loses strength when heated above $430{ }^{\circ} \mathrm{C}$ and is a poor conductor of heat and electricity. How to improve the thermal conductivity of titanium under the condition of ensuring the mechanical properties of titanium has become a key issue in engineering application research.

Due to outstanding properties, such as high thermal conductivity $\left(\sim 3000 \mathrm{Wm}^{-1}\right.$ $\left.\mathrm{K}^{-1}\right)$ [10,11], mechanical strength ( 125 GPa) [12,13], and tensile modulus ( 1.1 TPa) [14], graphene-metal materials exhibit high performance in thermal and mechanical properties and enhance the chemical stability of the metal [15-20]. For example, few-layer graphene (FLG)-reinforced copper composites were fabricated by spark plasma sintering (SPS) with an FLG volume fraction of $2.4 \mathrm{vol} \%$ [13]. The conductance of composites reached $70.4 \%$ of international annealed copper standard (IACS) [21,22]. Graphene-Cu nanocomposites foils were synthesized by an electrochemical method showed a high level of hardness, up to 2.2-2.5 GPa, and an elastic modulus of $137 \mathrm{GPa}$, which were increased by $96 \%$ and $30 \%$ 
from those of pure $\mathrm{Cu}$, respectively [12,23]. Graphene-Al composites were obtained by powder a metallurgy technique and exhibited greatly improved mechanical properties after adding graphene $[7,24,25]$. Therefore, the thermoelectric properties and mechanical stability of pure Ti and its alloys may be effectively enhanced by adding a certain amount of graphene.

The thermal conductivity of Ti-based metal-matrix composites increases with amount of graphene, as confirmed through the experiments in the previous studies [19,26-28]. $\mathrm{Gr} / \mathrm{Ti}$ composites outperform most carbon nanotube/Ti in thermal conductivity. Furthermore, the thermal conductivity and specific heat capacity of the composite material increase drastically with the increase of the graphene content [29,30]. Zheng et al. [31] reported that when 8 atomic layers of graphene were affiliated to the $\mathrm{Gr} / \mathrm{Ti}$ composites, the interface thermal conductivity was as high as $440 \mathrm{MW} / \mathrm{m}^{2} \mathrm{~K}$. However, there is still a vacuum in the research on the physical mechanism behind this phenomenon.

In this work, we study the thermal boundary conductance at the $\mathrm{Ti} / \mathrm{Gr}$ interface using both non-equilibrium molecular dynamics (NEMD) and thermal relaxation (TR) methods. The NEMD simulation is helpful in understanding the thermal conductivity of the composite and the TR method mimics the experimental laser-based pump-probe process. The relationship between the thermal boundary conductance and the phonon spectra at the interface is discussed in great detail. Our work suggests that the thermal properties of titanium can be greatly improved by affiliating an appropriate amount of graphene. In addition, compressive strain is an effective method to enhance the TBC of the Ti/Gr interface.

\section{Materials and Methods}

Ti is a kind of metal with hexagonal closest packed (HCP) structure. We chose three of the most common crystal faces, $\mathrm{Ti}(10-10), \mathrm{Ti}(11-20)$, and $\mathrm{Ti}(0001)$, as shown in Figure 1a, to build the model of $\mathrm{Ti} / \mathrm{Gr}$ interfaces. In order to establish the Ti/Gr interface structure with minimal lattice mismatch, we selected different lengths for the titanium and graphene models for the corresponding crystal faces. Table 1 lists the dimensions of the models in the $\mathrm{x}$-direction and $\mathrm{y}$-direction, as well as the strain rate of graphene in each direction, where graphene sheets were subjected to a tensile strain at both the $\mathrm{x}$ - and $\mathrm{y}$-directions with $\mathrm{Ti}$ blocks keeping strain-free. The thickness of the titanium in the z-direction was about $2 \mathrm{~nm}$.

Table 1. The simulation parameters of the model with different crystal faces of titanium and the mismatch strain of graphene.

\begin{tabular}{cccccc}
\hline Crystal Face & $\mathbf{L}_{\mathbf{x}}(\mathbf{n m})$ & $\mathcal{E}_{\mathbf{G r}-\mathbf{x}}$ & $\mathbf{L}_{\mathbf{y}}(\mathbf{n m})$ & $\mathcal{E}_{\mathbf{G r}-\mathbf{y}}$ & $\mathbf{L}_{\mathbf{z}}(\mathbf{n m})$ \\
\hline $\operatorname{Ti}(10-10)$ & 7.2272 & $0.204 \%$ & 6.6435 & $0.0429 \%$ & 2.0 \\
$\operatorname{Ti}(11-20)$ & 7.2272 & $0.204 \%$ & 5.901 & $0.0305 \%$ & 1.98 \\
$\operatorname{Ti}(0001)$ & 5.1104 & $0.0310 \%$ & 5.901 & $0.0305 \%$ & 1.959 \\
\hline
\end{tabular}

All molecular dynamics (MD) simulations were performed using the large-scale atomic/molecular massively parallel simulator (LAMMPS) package [32]. The modified embedded-atom method (MEAM) potential [33] was employed to describe the atomic interactions in titanium, while the interactions between carbon atoms were described by the optimized Tersoff potential [34]. The van der Waals interactions of interlayers were modeled with Lennard-Jones (LJ) function $\mathrm{V}\left(r_{i j}\right)=4 \varepsilon\left[\left(\sigma / r_{i j}\right)^{12}-\left(\sigma / r_{i j}\right)^{6}\right]$ and the corresponding parameters are shown as $\sigma_{\mathrm{Ti}-\mathrm{Gr}}=0.36 \mathrm{~nm}$ and $\varepsilon_{\mathrm{Ti}-\mathrm{Gr}}=0.006535 \mathrm{eV}$ [35]. The time step was set as $0.5 \mathrm{fs}$. The cutoff distance of the LJ potential was set as $1.2 \mathrm{~nm}$. The non-periodic boundary conditions (Figure $1 \mathrm{~b}, \mathrm{c}$ ) were employed to explore the TBC at the $\mathrm{Ti} / \mathrm{Gr}$ interface for both the NEMD and thermal relaxation methods. 
(a)

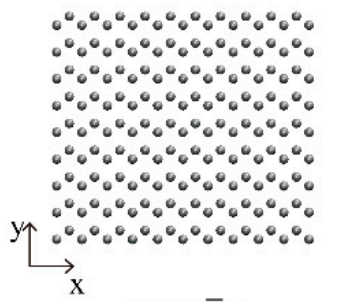

$\operatorname{Ti}(10 \overline{1} 0)$

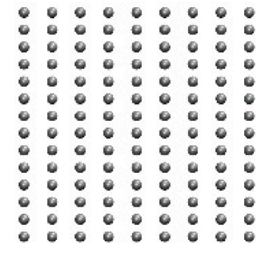

$\operatorname{Ti}(11 \overline{2} 0)$

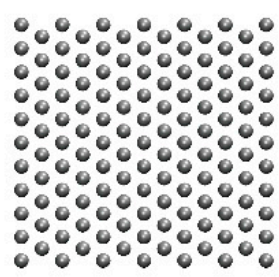

$\operatorname{Ti}(0001)$ (b)

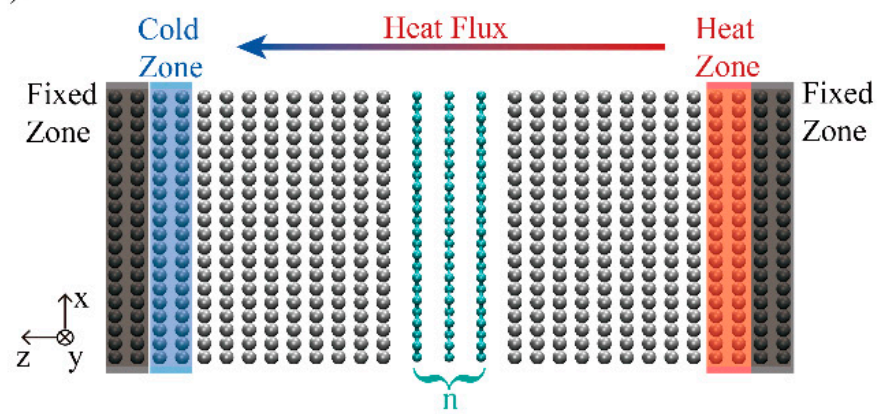

(c)

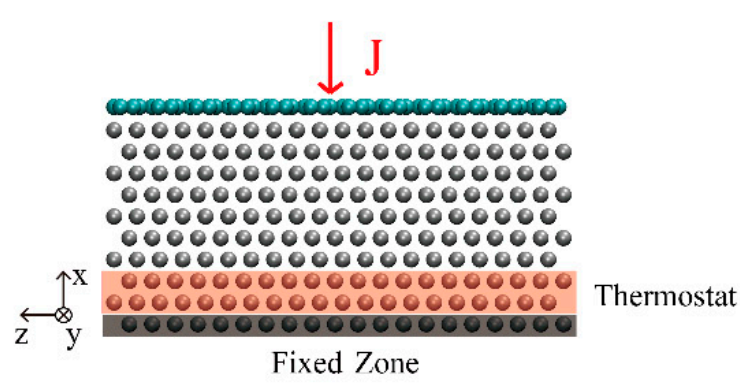

Figure 1. (a) Atomic structure of the first layer lattice and structure snapshots of the interfacial Ti layer for the (10-10), (11-20), and (0001) surfaces. (b) The schematic model setup for the NEMD method. (c) The MD model of the Ti/Gr interface under free boundary condition using the thermal relaxation method.

\subsection{NEMD Method}

To calculate the TBC based on the NEMD, simulations were conducted using the nonperiodic boundary condition model (Figure 1b), in which the periodic boundary conditions were applied along the in-plane ( $\mathrm{x}$ and $\mathrm{y}$ ) directions, with the free-boundary condition along the out-of-plane $(\mathrm{z})$ direction. We set the heat zone and the cold zone at the temperatures of $340 \mathrm{~K}$ and $260 \mathrm{~K}$ by using the Langevin thermostat [36]. The system occurred a directional thermal transfer process because of the temperature difference. After $0.5 \mathrm{~ns}$, a temperature gradient was generated and each part was time-independent. Any block of the Ti was divided into 25 equal slabs along the heat flux direction $(z)$ to calculate the temperature distribution, and the last 3 ns of data was collected. Heat flux is the change in energy over time: $\mathrm{J}=\mathrm{d} \Delta \mathrm{E}(\mathrm{t}) / \mathrm{dt}$, where $\Delta \mathrm{E}$ represents the energy change. $\mathrm{TBC}$ can be calculated as follows: $G=J / A \Delta T$, where $A$ is the cross-section of heat transfer. Herein, we take the value of $\Delta \mathrm{T}$ by averaging both of the two-temperature differences that occurred close to the heat source and the heat sink of the Ti/Gr interfaces.

\subsection{Thermal Relaxation Method}

In the TR studies, the non-periodic boundary condition model in the surface-normal direction, as shown in Figure 1c, was employed. A time step of $0.5 \mathrm{fs}$ was used in the MD simulations. First, the initial configuration was equilibrated at $300 \mathrm{~K}$ by performing a constant volume and a constant temperature (NVT) for $1.0 \mathrm{~ns}\left(2 \times 10^{6}\right.$ steps $)$ to relax the structure of the system and to optimize the interlayer distance. Afterwards, the system was then switched to the microcanonical ensemble (NVE). The initial temperature gap could be generated by increasing the temperature of the graphene layers instantaneously to a specified value by rescaling the velocities of the carbon atoms [37]. After the heat source was removed, the temperature of the graphene layer decayed exponentially within a few hundred picoseconds. The temperature profile of the graphene, as shown in Figure 2, was fitted by an exponential function and the relaxation time $\tau$ was obtained using $\Delta \mathrm{T}=\Delta \mathrm{T}\left(t_{0}\right) \exp \left(\left(t_{0}-\mathrm{t}\right) / \tau\right)$. Then, the value of the TBC was calculated via $\mathrm{G}=C /(A \times \tau)$, where $\mathrm{C}$ is the heat capacity of graphene. The heat capacity, $\mathrm{C}$, of graphene was computed, using the molecular dynamics approach, as a function of temper- 
ature $[38,39]$. The calculation was performed by varying the temperature in $5 \mathrm{~K}$ increments over a $\pm 10 \mathrm{~K}$ range around the temperature of interest. For instance, the total energy, E, was computed at $\mathrm{T}=290,295,300,305$, and $310 \mathrm{~K}$. The results were averaged over $1.5 \times 10^{5}$ steps under five independent simulations. The slope of a linear fitting is the heat capacity at the interest temperature.

(a)

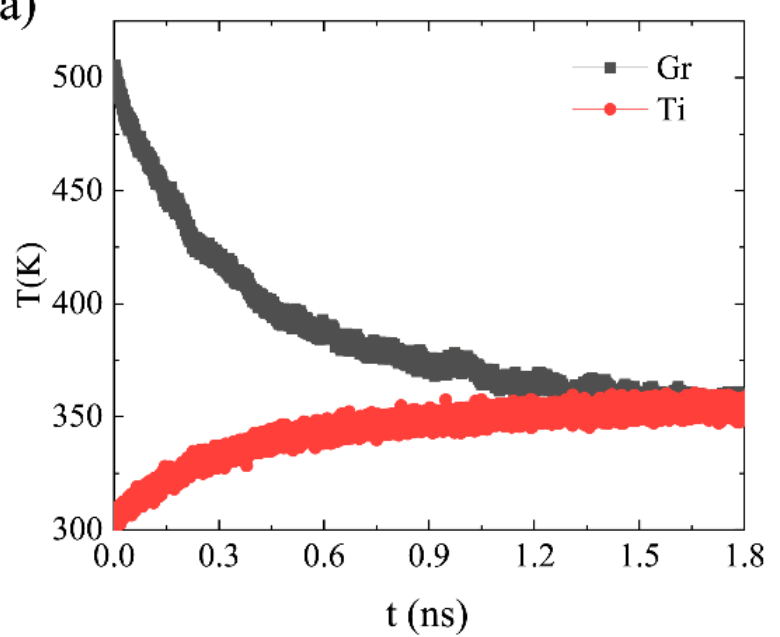

(b)

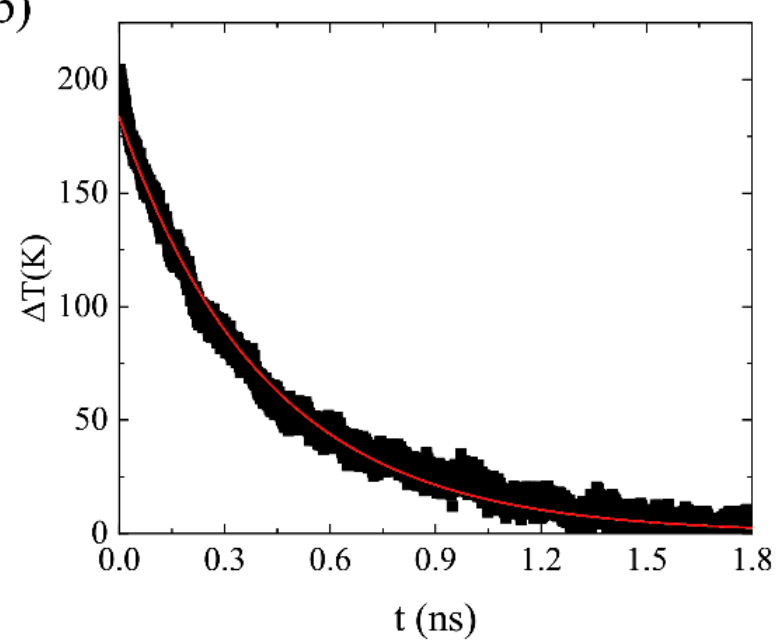

Figure 2. (a) Profile of the temperature evolution in the graphene sheets (black line) and the bulk of the Ti (red line) using the thermal relaxation method. (b) Profile and exponential fitting curves of $\Delta \mathrm{T}$.

\subsection{Phonon Spectra}

To elucidate the heat transfer mechanism across the interface, we investigated the vibrational density of states (VDOS) at the interfaces obtained from the Fourier transform of the interfacial atoms' velocity autocorrelation [40].

$$
\mathrm{P}(\omega)=\frac{1}{\sqrt{2 \pi}} \int_{0}^{\infty} e^{i \omega t}<\sum_{j=1}^{N} v_{j}(t) v_{j}(0)>d t
$$

The thermal transport between graphene and $\mathrm{Ti}$ is mainly dominated by the phonon in the out-of-plane direction, and most of the phonon coupling is contributed by the low-frequency zone [41]. The overlap of the phonon spectrum parameter, $\mathrm{S}$, is defined as:

$$
\mathrm{S}=\frac{\int_{0}^{\infty} P_{G r}(\omega) P_{T i}(\omega) d \omega}{\int_{0}^{\infty} P_{G r}(\omega) d \omega \int_{0}^{\infty} P_{T i}(\omega) d \omega}
$$

where $P_{G r}(\omega)$ and $P_{T i}(\omega)$ denote the phonon spectra at frequency $\omega$ of carbon and Ti atoms at the interface, respectively.

\section{Results and Discussion}

\subsection{Thermal Boundary Conductance at the Ti/Gr Interface}

We first compared the TBC results obtained from both two methods, as shown in Figure 3. The TBC of $\mathrm{Ti}(10-10) / \mathrm{Gr}$ exhibited the worst performance and $\mathrm{Ti}(0001) / \mathrm{Gr}$ showed the best, as illustrated in Figure 3a. Here we normalized the TBC value of Ti(0001)graphene interface as 1.0 to obtain the tendency of the TBC. The results show that the TBC obtained by the two methods was almost completely consistent with the variety of the crystal faces. The (0001) surface had the highest TBC, followed by the (11-20) surface. The (10-10) surface had the lowest TBC, indicating the robustness of the simulations. Although, the TBC of NEMD about the Ti(0001)-graphene interface was $425 \mathrm{MW} / \mathrm{m}^{2} \mathrm{~K}$, while the TBC of the thermal relaxation methods was $161 \mathrm{MW} / \mathrm{m}^{2} \mathrm{~K}$. To elucidate the inner mechanism of the TBC diversity obtained from both the NEMD and TR methods, we calculated the 
phonon spectrum and the phonon coupling parameter, as shown in Figure 3b,c. The phonons in Figure $3 \mathrm{~b}$ mainly distributed in the range of $1-30 \mathrm{THz}$, while Figure $3 \mathrm{c}$ shows that phonons only existed in the interval of $1-40 \mathrm{THz}$. The NEMD method can form a stable heat source and temperature gradient, including a higher frequency of phonons participating in interface heat conduction. For the thermal relaxation method, the initial high temperature brings about mostly high-frequency phonons, while the low-frequency phonons play a major role in the TBC, which results in the thermal relaxation method providing lower measurements than the NEMD method. This is also consistent with the previously reported results $[42,43]$.

(a)

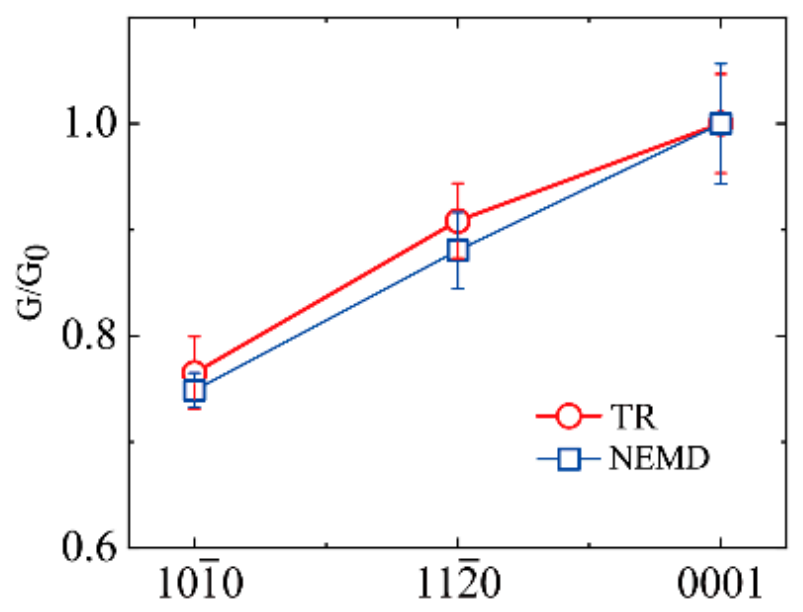

(b)

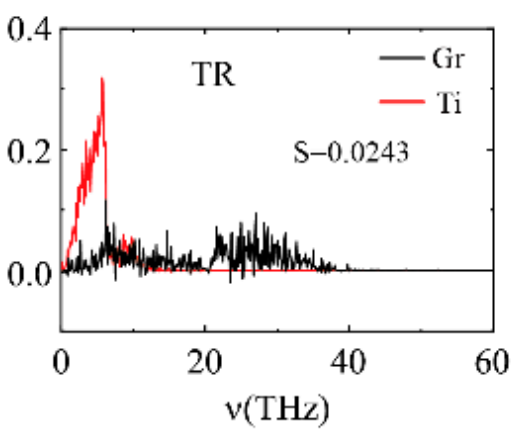

(c)

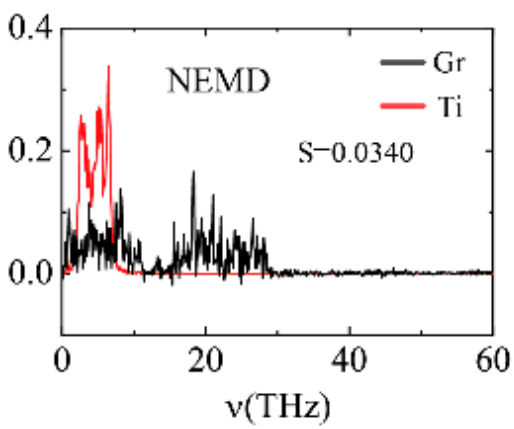

Figure 3. (a) The thermal boundary conductance obtained by the two methods, NEMD and TR, varied with the crystal faces of Ti. $\mathrm{G}_{0}$ is the TBC value of $\mathrm{Ti}(0001) / \mathrm{Gr}$. $(\mathbf{b}, \mathbf{c})$ The out-of-plane atomic vibration spectra of the Ti(0001)-graphene interface obtained by the NEMD (b) and TR (c) methods, respectively.

In addition, we analyzed the relationship between the phonon coupling strength and the TBC of different crystal faces. Since the results of NEMD were consistent with the thermal relaxation method, here we computed the TBC with different crystal faces using the TR method in Figure 4. The phonon spectra of the different crystal faces are illustrated in Figure $4 a-c$. The distribution range of phonons is almost the same, with only a slight difference in intensity in the low-frequency coupling range, which is the main reason for the diversity in the TBC (Figure 4d). Therefore, the choice of different crystal faces has a prominent influence on the heat transfer effect.

\subsection{Temperature Effects}

We further explored the dependence of $\mathrm{G}$ on the environmental temperature, $\mathrm{T}$, using the NEMD method for precise temperature maintenance and easy control. As shown in Figure 5, the TBC gradually increases with temperature, from $409 \mathrm{MW} / \mathrm{m}^{2} \mathrm{~K}$ at $250 \mathrm{~K}$ to $502 \mathrm{MW} / \mathrm{m}^{2} \mathrm{~K}$ at $450 \mathrm{~K}$. It features an almost linear dependence relationship (the fitted linear equation is: $\mathrm{G}=\mathrm{aT}+\mathrm{b}$, here $\mathrm{a}=0.48$ and $\mathrm{b}=287.36$ ) for the Umklapp processes, which play the dominant role in heat conduction [44]. Correspondingly, the overlapping factor, $\mathrm{S}$, also increases linearly with temperature. Furthermore, the representative phonon 
spectra are illustrated in Figure 6 to obtain insight into the heat transfer mechanism of the TBC of Ti/Gr interface.

(a)

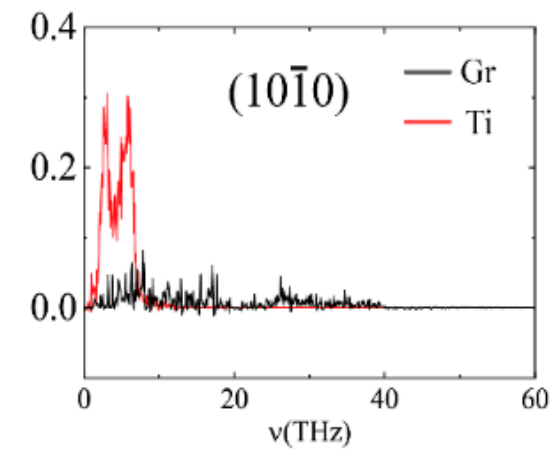

(c)

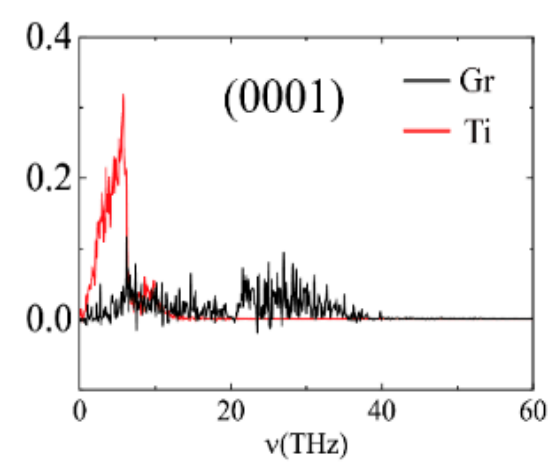

(b)

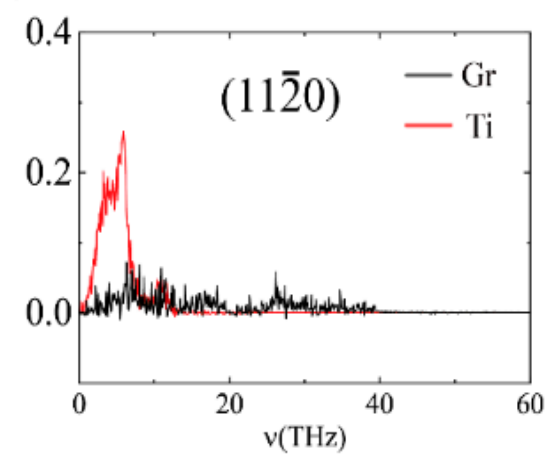

(d)

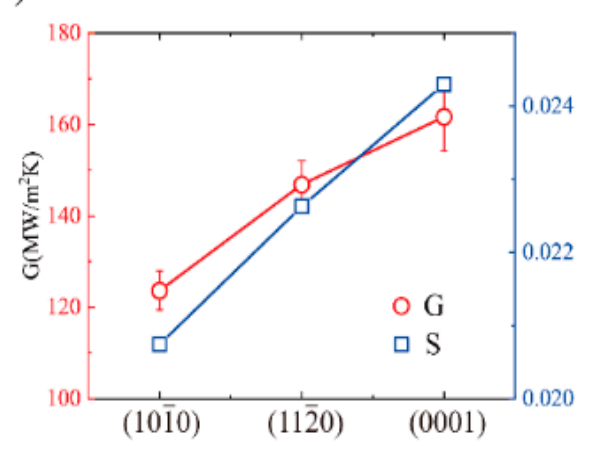

Figure 4. Thermal interface conductance and the out-of-plane phonon spectra of different crystal faces obtained by the heat dissipation method: (a) the phonon spectra of the Ti(10-10)-graphene interface; (b) the phonon spectra of the Ti(11-20)-graphene interface; (c) the phonon spectra of the Ti(0001)-graphene interface; and (d) the TBC and phonon coupling strength of the Ti/Gr interface.

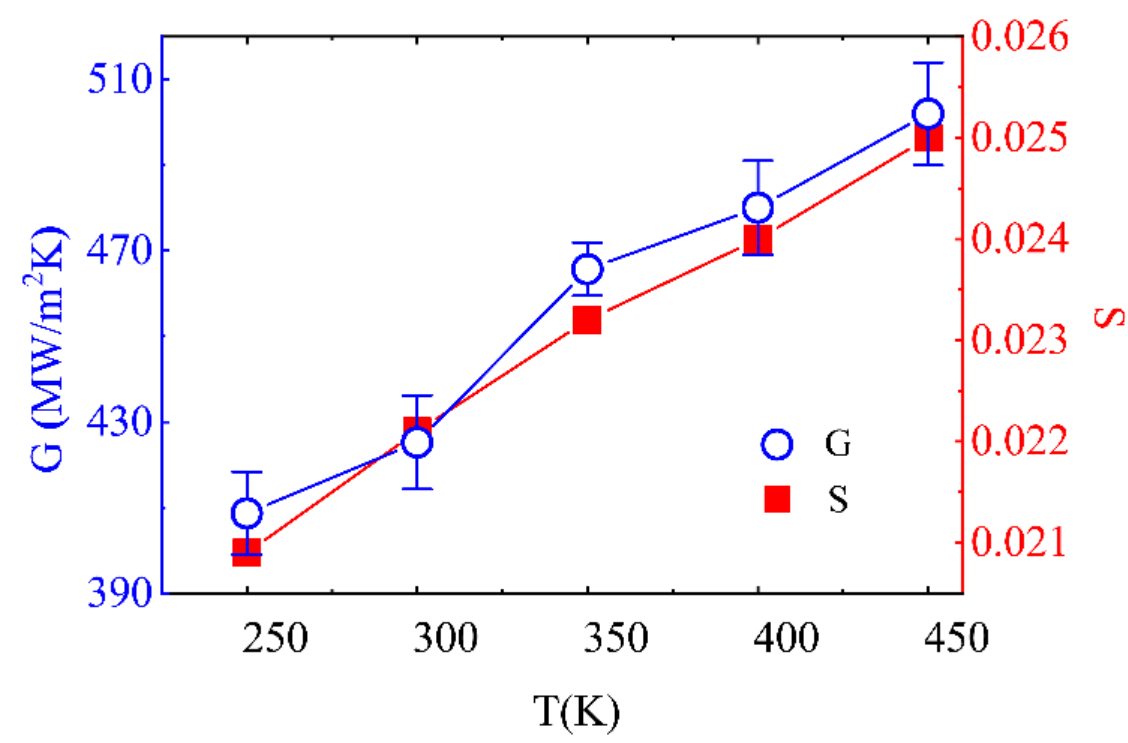

Figure 5. Varying range of the thermal boundary conductance and phonon coupling strength, S, along with temperature.

Comparing the in-plane and out-of-plane phonon spectra with the different temperatures in Figure 6, there is an interesting phenomenon that both the in-plane and out-of-plane phonon spectra of Ti had almost the same frequency and peak distributions, while the 
graphene had an enormous difference. This is attributed to the fact that graphene is a twodimensional material, exhibiting enormous anisotropic properties between the in-plane and out-of-plane directions. Since the phonon spectra of Ti were almost all distributed in the low frequency region (1-10 THz), out-of-plane phonons dominated the TBC, and its overlapped parameter $S$ was about one order higher than that of the in-plane phonon overlap $S$ value. The phonon coupling strength was enhanced at the out-of-plane components, and the corresponding $S$ values increased from 0.0318 to 0.0376 with the temperature.

(a1)

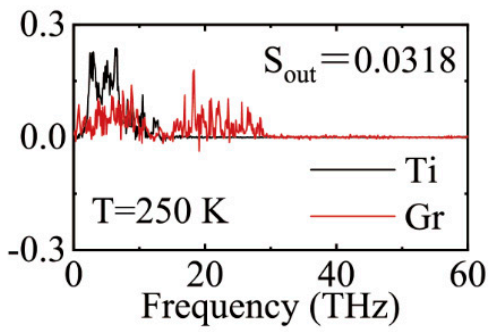

(a2)

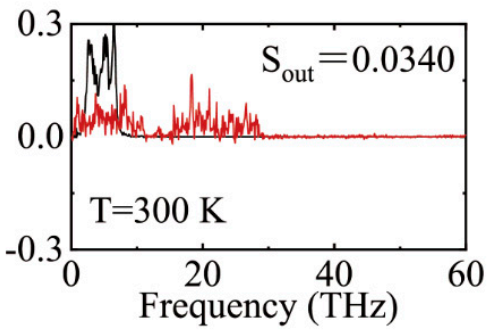

(a3)

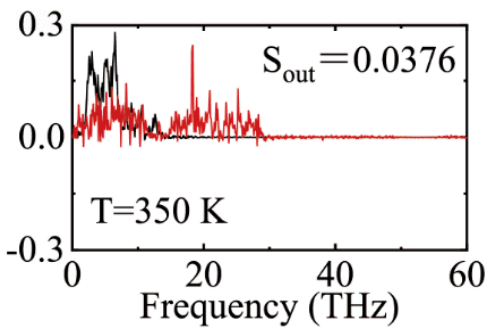

(a4)

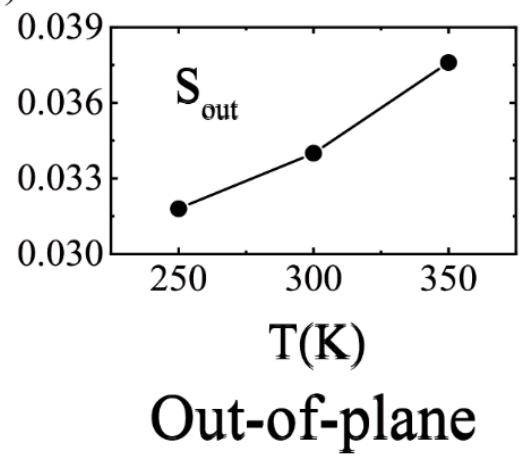

(b1)

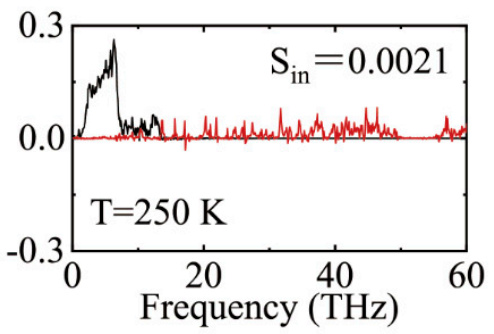

(b2)

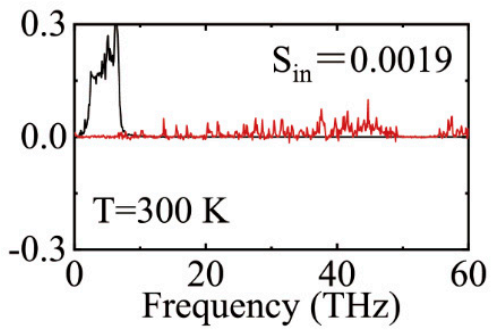

(b3)

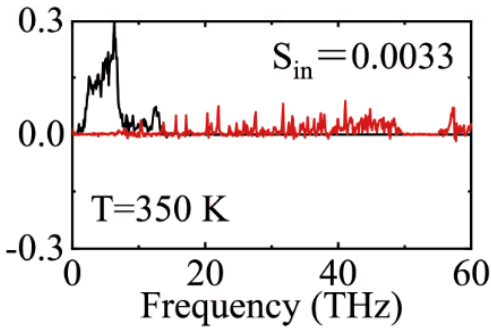

(b4)

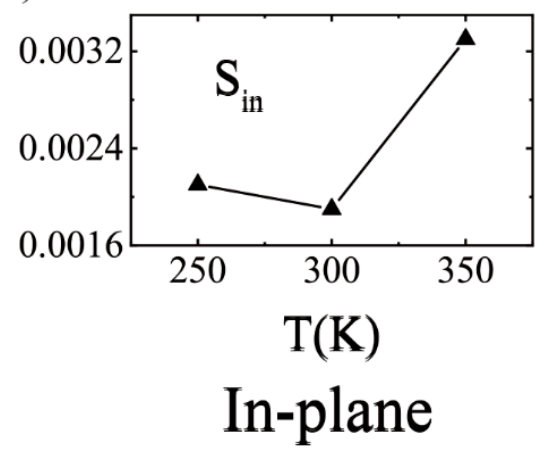

Figure 6. (a1-a3,b1-b3) Vibration density of states of atoms in interfaces of Ti and Gr under various temperatures. $(\mathbf{a} 4, \mathbf{b} 4)$ the overlap parameter $S$ of out-of-plane and in-plane dimensions, respectively.

\subsection{Size Effect of Graphene}

We studied the TBC variation with graphene layers, N. The layer number of a graphene sheet can be controlled by CVD growth or physical translation. Here, the interfacial model of $\mathrm{Ti}(0001) / \mathrm{Gr}$ model was employed and $\mathrm{n}$ represents the number of graphene layers, with the consideration of a few layers of graphene stacking in the experiment. The simulations were carried out by the NEMD method at $300 \mathrm{~K}$, and the corresponding schematic model is shown in Figure 7. Both ends were fixed with cold (heat sink) and hot (heat source) regions neighboring fixed regions. As shown in Figure $7 b$, the TBC decreased with an increasing $n$ 
until it converged at $n=5$. There was a reduction of $85 \%$ from $425 \pm 11 \mathrm{MW} / \mathrm{m}^{2} \mathrm{~K}(\mathrm{n}=1)$ to $63 \pm 1 \mathrm{MW} / \mathrm{m}^{2} \mathrm{~K}(\mathrm{n}=5)$. This was due to the increasing thickness of graphene block, which enhanced the interfacial phonon scattering at the interface. Similar phenomena have been reported in heat transport across the graphene/ $\mathrm{Cu}$ and graphene/Ni interfaces $[45,46]$. To explain this phenomenon, the corresponding in-plane phonon and out-of-plane coupling strength are illustrated in Figure $7 \mathrm{c}, \mathrm{d}$, respectively. The in-plane phonon coupling strength were almost independent of $\mathrm{n}$. It was, apparently, not the reason for the reduction of the TBC. The out-of-plane phonon coupling strength monotonically decreased with the number of layers, and the corresponding overlapping factor S dropped from $0.034(n=1)$ to 0.020 $(n=9)$, which is consistent with the trend of the TBC. Figure 7 shows that the magnitude of overlap factor $S$ in the out-of-plane direction was about one order higher than that of the in-plane direction. From the analysis of the previous results, the out-of-plane phonons of graphene play a dominant role on the TBC.

(a)

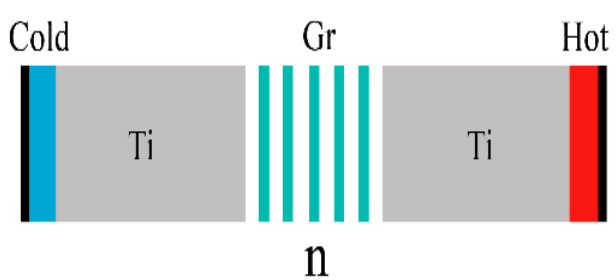

(c)

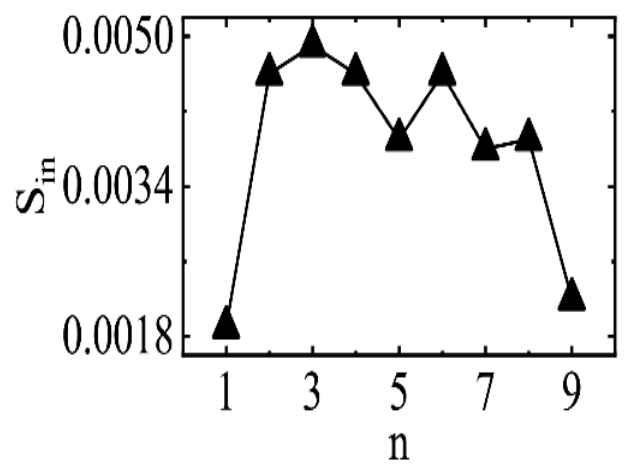

(b)

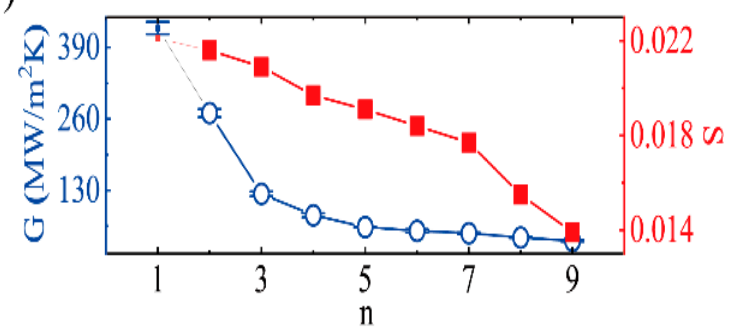

(d)

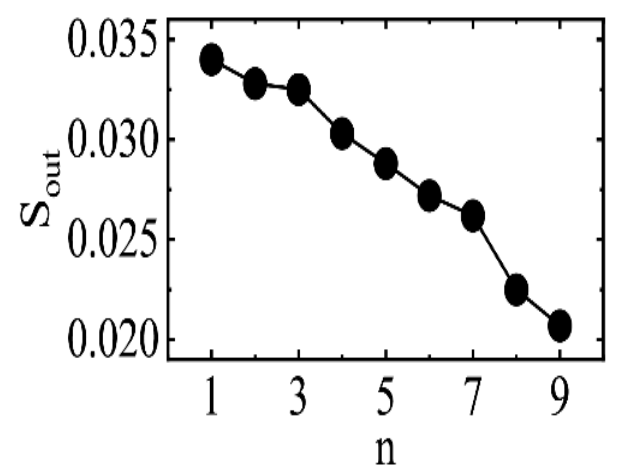

Figure 7. (a) The schematic of the thermal boundary conductance with the size effect of graphene. $n$ represents the number of graphene layers. (b) Varying range of the thermal boundary conductance and phonon coupling strength, $S$, along with $n$. (c) Varying range of the in-plane phonon coupling strength, $\mathrm{S}$, along with $\mathrm{n}$. (d) Varying range of the out-of-plane phonon coupling strength, $\mathrm{S}$, along with $\mathrm{n}$.

\subsection{Effect of Strain Engineering}

The thermal conductivity and the TBC are sensitive to the conducted strain. Applying a cross-face strain is an effective method to tune the heat transport across the interface, as confirmed in the previous studies of graphene [47], $\mathrm{MoS}_{2}$ [48], and black phosphorus [49]. Here, we performed a surface-normal strain along the direction of the TBC using the NEMD model. The uniaxial strain was applied by adjusting the $\mathrm{d}$ (distance) between two bulks of Ti. As illustrated in Figure 8a, the strain changes from tension to compression (3--3\%). The graphene sheet sandwiched between two Ti bulks transformed from a state full of ripples to a flat state. The stress-strain relationship and the distance between the two bulks of $\mathrm{Ti}, \mathrm{d}$, with conducted strain are depicted in Figure $8 \mathrm{~b}$. When the strain was about $-6 \%$, the stress was approximately $120 \mathrm{GPa}$ and the graphene structure broke at such a high 
pressure. Thus, we only studied the strain range from $-3 \%$ to $3 \%$. The block interlayer distance, $d$, increased monotonously with increasing strain in Figure 8 b.
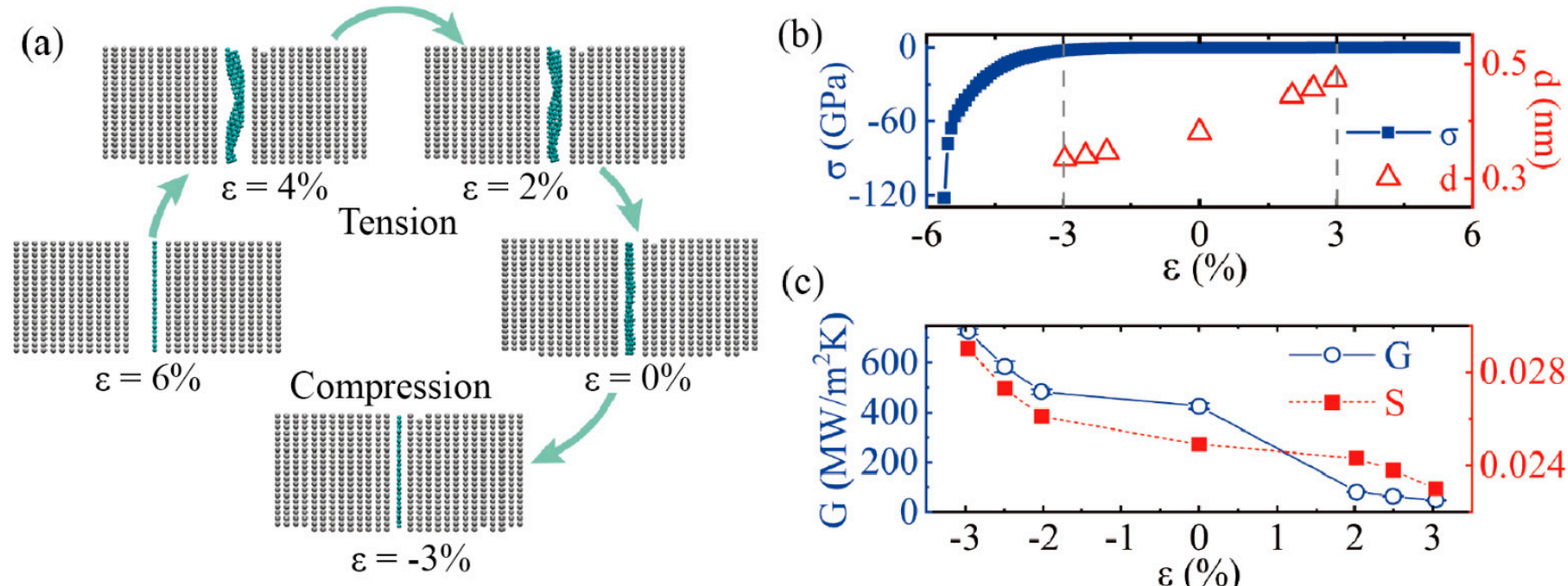

(c)

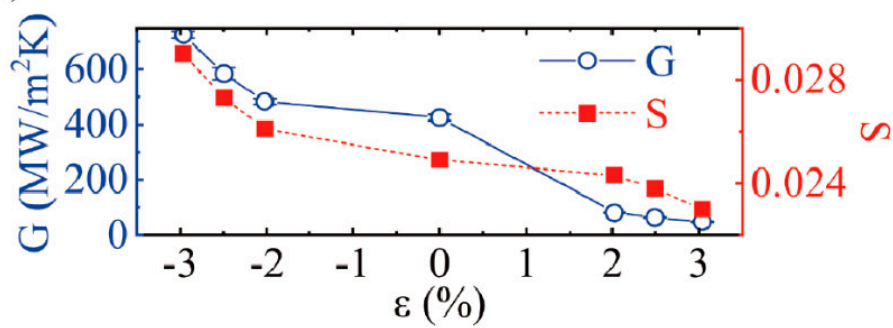

Figure 8. (a) The Snapshots of Ti and graphene under different strains. (b) Varying range of the stress and distance between two bulks of Ti, along with strain. (c) Varying range of the thermal boundary conductance and phonon coupling strength, $\mathrm{S}$, along with strain.

The compressive strain was found to enhance the thermal conductance, G, and followed an exponential dependence relationship, which is consistent with previous studies $[42,48]$. In Figure $8 c$, when the strain rate was $-3 \%$, the TBC was about $700 \mathrm{MW} / \mathrm{m}^{2} \mathrm{~K}$, which is almost twice as high as the TBC in the strain-free state. However, the TBC was only about $48 \mathrm{MW} / \mathrm{m}^{2} \mathrm{~K}$ with the strain at $3 \%$. The enormous TBC difference indicates that applying compressive strain to Ti and graphene is an efficient way to improve the TBC. In order to elucidate the strain effect on the TBC, we investigated the variation of phonon coupling strength under different strains. As shown in Figure 8c, the $S$ was about 0.0299 with the compressive strain rate at $-3 \%$, while it was about 0.0173 with the tensile strain rate at $3 \%$. There was a satisfying agreement between the overlapping factor, $\mathrm{S}$, and the interface thermal conductivity with the strain rate. These findings are consistent with the above conclusions. Hence, the TBC can be effectively modulated by cross-face strain. The thermal conductivity of $\mathrm{Ti} / \mathrm{Gr}$ MMCs is computed taking into account TBC effects at the $\mathrm{Ti} / \mathrm{Gr}$ interfaces, and the thermal conductivity increases with graphene volume content. Our findings also provide insights into ways to optimize future thermal management based on MMC materials.

\section{Conclusions}

In summary, the thermal boundary conductance at $\mathrm{Ti} / \mathrm{Gr}$ interfaces was investigated systematically via MD simulations. Among the $\mathrm{Ti}(0001) / \mathrm{Gr}$, $\mathrm{Ti}(10-10) / \mathrm{Gr}$, and $\mathrm{Ti}(11-20) / \mathrm{Gr}$ models, the $\mathrm{Ti}(0001) / \mathrm{Gr}$ model exhibited the best performance of TBC at the interface and $\mathrm{Ti}(10-10) / G r$ showed the lowest TBC value. The TBC increased with increasing temperature and its value increased $18.1 \%$ when the background temperature raised from $300 \mathrm{~K}$ to $450 \mathrm{~K}$. The TBC decreased with increasing interfacial graphene layer numbers. A reduction of $85 \%$ was reported for an n-layer $(n \geq 5)$ graphene block compared with the case of a single-layer graphene interface. Our results exhibit enormous potential of Ti/Gr MMCs in thermal management and cooling applications.

Author Contributions: Conceptualization, B.O. and L.L.; methodology, B.O.; validation, B.O. and L.L.; formal analysis, J.Y.; investigation, J.Y.; resources, Q.W.; data curation, J.Y.; writing-original draft preparation, B.O.; writing-review and editing, B.O.; project administration, L.L.; funding acquisition, Q.W. All authors have read and agreed to the published version of the manuscript. 
Funding: This research was funded by the science and technology project of the Jiangsu Provincial Bureau of market supervision, grant number KJ21125038, and the science and technology project of the Special Equipment Safety Supervision Inspection Institute of Jiangsu Province, grant number $\mathrm{KJ}(\mathrm{Y}) 2020038$.

Institutional Review Board Statement: Not applicable.

Informed Consent Statement: Not applicable.

Data Availability Statement: The authors can confirm that all relevant data are included in the article.

Acknowledgments: The authors would like to acknowledge the support provided by Jiangsu Key Laboratory of Advanced Food Manufacturing Equipment and Technology, Jiangnan University and by the Special Equipment Safety Supervision Inspection Institute of Jiangsu Province, National Graphene Products Quality Inspection and Testing Center (Jiangsu) for the facilities necessary to implement the experimental research. Additionally, the authors would like to thank Qing sheng Wang ("National Graphene Products Quality Inspection and Testing Center (Jiangsu)) for providing the resources.

Conflicts of Interest: The author declares no conflict of interest.

\section{References}

1. Banerjee, D.; Williams, J. Perspectives on titanium science and technology. Acta Mater. 2013, 61, 844-879. [CrossRef]

2. Fanning, J. Military applications for $\beta$ titanium alloys. J. Mater. Eng. Perform. 2005, 14, 686-690. [CrossRef]

3. Kang, X.; Liu, S.; Dai, Z.; He, Y.; Song, X.; Tan, Z. Titanium dioxide: From engineering to applications. Catalysts 2019,9 , 191. [CrossRef]

4. Kaur, M.; Singh, K. Review on titanium and titanium based alloys as biomaterials for orthopaedic applications. Mater. Sci. Eng. C 2019, 102, 844-862. [CrossRef]

5. Kim, K.T.; Eo, M.Y.; Nguyen, T.T.H.; Kim, S.M. General review of titanium toxicity. Int. J. Implant Dent. 2019, 5, 1-12. [CrossRef]

6. Piątek, A.; Nowak, R.; Gburski, Z. A Titanium-Decorated Fullerene Cluster-A Molecular Dynamics Simulation. Solid State Phenom. 2008, 140, 109-116. [CrossRef]

7. Yan, Q.; Chen, B.; Li, J. Super-high-strength graphene/titanium composites fabricated by selective laser melting. Carbon 2021, 174, 451-462. [CrossRef]

8. Casillas, N.; Charlebois, S.; Smyrl, W.H.; White, H.S. Pitting Corrosion of Titanium. J. Electrochem. Soc. 1994, 141, 636-642. [CrossRef]

9. Kaseem, M.; Choe, H.-C. Simultaneous improvement of corrosion resistance and bioactivity of a titanium alloy via wet and dry plasma treatments. J. Alloys Compd. 2021, 851, 156840. [CrossRef]

10. Kotov, N.A. Carbon sheet solutions. Nature 2006, 442, 254-255. [CrossRef]

11. Wei, N.; Chen, Y.; Cai, K.; Zhang, Y.; Pei, Q.; Zheng, J.-C.; Mai, Y.-W.; Zhao, J. Unusual thermal properties of graphene origami crease: A molecular dynamics study. Green Energy Environ. 2022, 7, 86-94. [CrossRef]

12. Konatham, D.; Striolo, A. Thermal boundary resistance at the graphene-oil interface. Appl. Phys. Lett. 2009, 95, 163105. [CrossRef]

13. Chen, L.; Huang, Z.; Kumar, S. Impact of bonding at multi-layer graphene/metal Interfaces on thermal boundary conductance. RSC Adv. 2014, 4, 35852-35861. [CrossRef]

14. Geim, A.K.; Novoselov, K.S. The rise of graphene. Nat. Mater. 2007, 6, 183-191. [CrossRef]

15. Fonseca, A.F.; Liang, T.; Zhang, D.; Choudhary, K.; Phillpot, S.R.; Sinnott, S.B. Graphene-Titanium Interfaces from Molecular Dynamics Simulations. ACS Appl. Mater. Interfaces 2017, 9, 33288-33297. [CrossRef]

16. Rafiee, M.A.; Rafiee, J.; Wang, Z.; Song, H.; Yu, Z.-Z.; Koratkar, N. Enhanced mechanical properties of nanocomposites at low graphene content. ACS Nano 2009, 3, 3884-3890. [CrossRef]

17. Dominguez, H.; Goicochea, A.G.; Mendoza, N.; Alejandre, J. Computer simulations of surfactant monolayers at solid walls. J. Colloid Interface Sci. 2006, 297, 370-373. [CrossRef]

18. Nazeer, F.; Ma, Z.; Gao, L.; Malik, A.; Khan, M.A.; Abrar, S.; Ahmed, B.; Wang, F.; Li, H. Effect of graphene on thermal and mechanical properties of copper-titanium carbide composites. Vacuum 2020, 173, 109100. [CrossRef]

19. Kanjwal, M.A.; Leung, W.W.-F. Titanium based composite-graphene nanofibers as high-performance photocatalyst for formaldehyde gas purification. Ceram. Int. 2019, 45, 5617-5626. [CrossRef]

20. Zhang, Y.-H.; Yue, L.-J.; Han, L.-F.; Chen, J.-L.; Jia, D.-Z.; Li, F. Tuning the magnetic and transport property of graphene with Ti atom and cluster. Comput. Mater. Sci. 2012, 56, 95-99. [CrossRef]

21. Schmidt, A.J.; Collins, K.C.; Minnich, A.J.; Chen, G. Thermal conductance and phonon transmissivity of metal-graphite interfaces. J. Appl. Phys. 2010, 107, 104907. [CrossRef]

22. Jagannadham, K. Thermal Conductivity Changes in Titanium-Graphene Composite upon Annealing. Metall. Mater. Trans. A 2015, 47, 907-915. [CrossRef] 
23. Kaushik, V.; Shukla, A.K.; Vankar, V.D. Improved electron field emission from metal grafted graphene composites. Carbon 2013, 62, 337-345. [CrossRef]

24. Zhou, T.; Zhao, Y.; Zhou, G.; Lv, W.; Sun, P.; Kang, F.; Li, B.; Yang, Q.-H. An in-plane heterostructure of graphene and titanium carbide for efficient polysulfide confinement. Nano Energy 2017, 39, 291-296. [CrossRef]

25. Yu, S.; Wei, D.; Shi, L.; Ai, Y.; Zhang, P.; Wang, X. Three-dimensional graphene/titanium dioxide composite for enhanced U (VI) capture: Insights from batch experiments, XPS spectroscopy and DFT calculation. Environ. Pollut. 2019, 251, 975-983. [CrossRef]

26. Yang, W.-Z.; Huang, W.-M.; Wang, Z.-F.; Shang, F.-J.; Huang, W.; Zhang, B.-Y. Thermal and Mechanical Properties of GrapheneTitanium Composites Synthesized by Microwave Sintering. Acta Metall. Sin. (Engl. Lett.) 2016, 29, 707-713. [CrossRef]

27. Han, J.; Yang, L.; Yang, L.; Jiang, W.; Luo, X.; Luo, S. PtRu nanoalloys loaded on graphene and TiO2 nanotubes co-modified Ti wire as an active and stable methanol oxidation electrocatalyst. Int. J. Hydrog. Energy 2018, 43, 7338-7346. [CrossRef]

28. Yang, J.; Ziade, E.; Maragliano, C.; Crowder, R.; Wang, X.; Stefancich, M.; Chiesa, M.; Swan, A.K.; Schmidt, A.J. Thermal conductance imaging of graphene contacts. J. Appl. Phys. 2014, 116, 023515. [CrossRef]

29. Yang, L.; Sun, L.; Bai, W.; Li, L. Thermal conductivity of Cu-Ti/diamond composites via spark plasma sintering. Diam. Relat. Mater. 2019, 94, 37-42. [CrossRef]

30. Zhang, Z.Y.; Zhu, Y.; Liang, Y.L. Preparation and thermal properties of graphene nanosheet/Ti composites. IOP Conf. Ser. Mater. Sci. Eng. 2017, 248, 012011. [CrossRef]

31. Zheng, H.; Jaganandham, K. Thermal Conductivity and Interface Thermal Conductance in Composites of Titanium with Graphene Platelets. J. Heat Transf. 2014, 136, 06130. [CrossRef]

32. Plimpton, S. Fast parallel algorithms for short-range molecular dynamics. J. Comput. Phys. 1995, 117, 1-19. [CrossRef]

33. Lenosky, T.J.; Sadigh, B.; Alonso, E.; Bulatov, V.V.; Rubia, T.D.d.l.; Kim, J.; Voter, A.F.; Kress, J.D. Highly optimized empirical potential model of silicon. Modell. Simul. Mater. Sci. Eng. 2000, 8, 825-841. [CrossRef]

34. Lindsay, L.; Broido, D.A. Optimized Tersoff and Brenner empirical potential parameters for lattice dynamics and phonon thermal transport in carbon nanotubes and graphene. Phys. Rev. B 2010, 81, 205441. [CrossRef]

35. Tang, W.; Zhang, J.; Wu, J.; Shao, J.; Ding, P.; Hou, G.; Chen, X. Mechanical properties and enhancement mechanisms of titanium-graphene nanocomposites. Acta Mech. Sin. 2020, 36, 855-865. [CrossRef]

36. Schneider, T.; Stoll, E. Molecular-dynamics study of a three-dimensional one-component model for distortive phase transitions. Phys. Rev. B 1978, 17, 1302. [CrossRef]

37. Jund, P.; Jullien, R. Molecular-dynamics calculation of the thermal conductivity of vitreous silica. Phys. Rev. B 1999, 59, 13707. [CrossRef]

38. Thomas, J.A.; Iutzi, R.M.; McGaughey, A.J. Thermal conductivity and phonon transport in empty and water-filled carbon nanotubes. Phys. Rev. B 2010, 81, 045413. [CrossRef]

39. Melis, C.; Colombo, L. Lattice thermal conductivity of Si 1- x Ge x nanocomposites. Phys. Rev. Lett. 2014, 112, 065901. [CrossRef]

40. Mollow, B.R. Power Spectrum of Light Scattered by Two-Level Systems. Phys. Rev. 1969, 188, 1969-1975. [CrossRef]

41. Lan, J.; Li, B. Thermal rectifying effect in two-dimensional anharmonic lattices. Phys. Rev. B 2006, 74, 214305. [CrossRef]

42. Chen, Y.; Zhang, Y.; Cai, K.; Jiang, J.; Zheng, J.-C.; Zhao, J.; Wei, N. Interfacial thermal conductance in graphene/black phosphorus heterogeneous structures. Carbon 2017, 117, 399-410. [CrossRef]

43. Li, S.; Chen, Y.; Zhao, J.; Wang, C.; Wei, N. Atomic structure causing an obvious difference in thermal conductance at the Pd-H2O interface: A molecular dynamics simulation. Nanoscale 2020, 12, 17870-17879. [CrossRef] [PubMed]

44. Wang, Y.; Xu, Z. The critical power to maintain thermally stable molecular junctions. Nat. Commun. 2014, 5, 4297. [CrossRef]

45. Chang, S.-W.; Nair, A.K.; Buehler, M.J. Geometry and temperature effects of the interfacial thermal conductance in copper-and nickel-graphene nanocomposites. J. Phys. Condens. Matter 2012, 24, 245301. [CrossRef]

46. Ghosh, S.; Bao, W.; Nika, D.L.; Subrina, S.; Pokatilov, E.P.; Lau, C.N.; Balandin, A.A. Dimensional crossover of thermal transport in few-layer graphene. Nat. Mater. 2010, 9, 555-558. [CrossRef]

47. Chen, J.; Walther, J.H.; Koumoutsakos, P. Strain Engineering of Kapitza Resistance in Few-Layer Graphene. Nano Lett. 2014, 14, 819-825. [CrossRef]

48. Ding, Z.; Pei, Q.-X.; Jiang, J.-W.; Huang, W.; Zhang, Y.-W. Interfacial thermal conductance in graphene/MoS2 heterostructures. Carbon 2016, 96, 888-896. [CrossRef]

49. Zhang, Y.-Y.; Pei, Q.-X.; Jiang, J.-W.; Wei, N.; Zhang, Y.-W. Thermal conductivities of single-and multi-layer phosphorene: A molecular dynamics study. Nanoscale 2016, 8, 483-491. [CrossRef] 